\title{
THE PUZZLING Li-RICH RED GIANT ASSOCIATED WITH NGC 6819
}

\author{
Joleen K. Carlberg ${ }^{1,22}$, Verne V. Smith ${ }^{2}$, Katia Cunha ${ }^{3,21}$, Steven R. Majewski ${ }^{4}$, Szabolcs Mészáros ${ }^{5,6}$, \\ Matthew Shetrone ${ }^{7}$, Carlos Allende Prieto ${ }^{8,9}$, Dmitry Bizyaev ${ }^{10,11}$, Keivan G. Stassun ${ }^{12,13}$, Scott W. Fleming ${ }^{14,15}$, \\ Gail Zasowski $^{16,23}$, Fred Hearty ${ }^{17}$, David L. Nidever ${ }^{18}$, Donald P. Schneider ${ }^{17,19}$, Jon A. Holtzman ${ }^{11}$, AND \\ Peter M. Frinchaboy 20 \\ ${ }^{1}$ Department of Terrestrial Magnetism, Carnegie Institution of Washington, 5241 Broad Branch Road, NW, Washington DC 20015, USA; \\ joleen.k.carlberg@nasa.gov \\ ${ }^{2}$ National Optical Astronomy Observatory, 950 North Cherry Avenue, Tucson, AZ 85719, USA \\ ${ }^{3}$ Observatório Nacional, Rua General José Cristino, 77, 20921-400 São Cristóvão, Rio de Janeiro, RJ, Brazil \\ ${ }^{4}$ Department of Astronomy, University of Virginia, Charlottesville, VA 22904, USA \\ ${ }^{5}$ ELTE Gothard Astrophysical Observatory, H-9704 Szombathely, Szent Imre herceg st. 112, Hungary \\ ${ }^{6}$ Department of Astronomy, Indiana University, Bloomington, IN 47405, USA \\ ${ }^{7}$ McDonald Observatory, University of Texas at Austin, TX 79734, USA \\ ${ }^{8}$ Instituto de Astrofísica de Canarias, C/Vía Láctea, s/n, E-38200, La Laguna, Tenerife, Spain \\ ${ }^{9}$ Departamento de Astrofísica, Universidad de La Laguna, E-38206, La Laguna, Tenerife, Spain \\ ${ }^{10}$ Apache Point Observatory, P.O. Box 59, Sunspot, NM 88349, USA \\ ${ }^{11}$ Department of Astronomy, MSC 4500, New Mexico State University, P.O. Box 30001, Las Cruces, NM 88003, USA \\ ${ }^{12}$ Department of Physics and Astronomy, Vanderbilt University, VU Station 1807, Nashville, TN 37235, USA \\ ${ }^{13}$ Department of Physics, Fisk University, 1000 17th Avenue North, Nashville, TN 37208, USA \\ ${ }_{15}$ Space Telescope Science Institute, 3700 San Martin Drive, Baltimore, MD, 21218 USA \\ ${ }^{15}$ Computer Sciences Corporation, 3700 San Martin Drive, Baltimore, MD, 21218 USA \\ ${ }^{16}$ Center for Astrophysical Sciences, Department of Physics and Astronomy, Johns Hopkins University, 3400 North Charles Street, Baltimore, MD 21218, USA \\ ${ }_{17}$ Department of Astronomy and Astrophysics, The Pennsylvania State University, 525 Davey Lab, University Park, PA 16802, USA \\ ${ }_{18}$ Department of Astronomy, University of Michigan, Ann Arbor, MI 48104, USA \\ ${ }^{19}$ Institute for Gravitation and the Cosmos, The Pennsylvania State University, University Park, PA 16802, USA \\ ${ }^{20}$ Department of Physics \& Astronomy, Texas Christian University, Fort Worth, TX 76129, USA \\ ${ }^{21}$ Steward Observatory, University of Arizona, Tucson, AZ 85719, USA \\ Received 2014 September 14; accepted 2015 January 19; published 2015 March 13
}

\begin{abstract}
A Li-rich red giant (RG) star (2M19411367+4003382) recently discovered in the direction of NGC 6819 belongs to the rare subset of Li-rich stars that have not yet evolved to the luminosity bump, an evolutionary stage where models predict $\mathrm{Li}$ can be replenished. The currently favored model to explain $\mathrm{Li}$ enhancement in first-ascent RGs like 2M19411367+4003382 requires deep mixing into the stellar interior. Testing this model requires a measurement of ${ }^{12} \mathrm{C} /{ }^{13} \mathrm{C}$, which is possible to obtain from Apache Point Observatory Galactic Evolution Experiment (APOGEE) spectra. However, the Li-rich star also has abnormal asteroseismic properties that call into question its membership in the cluster, even though its radial velocity and location on color-magnitude diagrams are consistent with membership. To address these puzzles, we have measured a wide array of abundances in the Lirich star and three comparison stars using spectra taken as part of the APOGEE survey to determine the degree of stellar mixing, address the question of membership, and measure the surface gravity. We confirm that the Li-rich star is a RG with the same overall chemistry as the other cluster giants. However, its $\log g$ is significantly lower, consistent with the asteroseismology results and suggestive of a very low mass if the star is indeed a cluster member. Regardless of the cluster membership, the ${ }^{12} \mathrm{C} /{ }^{13} \mathrm{C}$ and $\mathrm{C} / \mathrm{N}$ ratios of the Li-rich star are consistent with standard first dredge-up, indicating that Li dilution has already occurred, and inconsistent with internal $\mathrm{Li}$ enrichment scenarios that require deep mixing.
\end{abstract}

Key words: open clusters and associations: individual (NGC 6819) - stars: abundances - stars: chemically peculiar - stars: late-type

\section{INTRODUCTION}

Anthony-Twarog et al. (2013, hereafter AT13) recently reported the discovery of a Li-rich red giant $(\mathrm{RG})$ star in NGC 6819. This star, 2M19411367+4003382, has $A(\mathrm{Li}) \sim 2.3 \mathrm{dex}$ $([\mathrm{Li} / \mathrm{H}] \sim 1.3)$ and is unusual even within the class of rare $\mathrm{Li}-$ rich giants. The star's current position on a color-magnitude diagram (CMD) suggests that it has evolved beyond the $\mathrm{Li}$ dilution phase of first dredge-up (FDU), and, indeed, the other NGC 6819 RGs at similar magnitudes have $A(\mathrm{Li})<0.7$ dex (AT13). What makes this Li-rich star so unusual is that its

\footnotetext{
22 NASA Postdoctoral Program Fellow.

${ }^{23}$ NSF Astronomy and Astrophysics Postdoctoral Fellow.
}

CMD position is clearly below and blueward of the luminosity bump, the evolutionary stage where models have demonstrated that newly synthesized $\mathrm{Li}$ can be circulated to the stellar envelope, resulting in a brief stage of Li-richness (see, e.g., Charbonnel \& Balachandran 2000; Eggleton et al. 2008; Denissenkov 2012, hereafter D12). Other recent studies (e.g., Kumar et al. 2011 and Carlberg et al. 2012) have identified Lirich stars in the field that are too hot to be bump stars. The former study hypothesized that these stars are red clump (RC) stars that replenished $\mathrm{Li}$ during the $\mathrm{He}$ flash, and the low ${ }^{12} \mathrm{C} /{ }^{13} \mathrm{C}$ of many of these stars are consistent with internal $\mathrm{Li}$ regeneration. The latter study favored planetary engulfment because their stars tend to be more rapidly rotating and have ${ }^{12} \mathrm{C} /{ }^{13} \mathrm{C}$ near normal FDU values. 

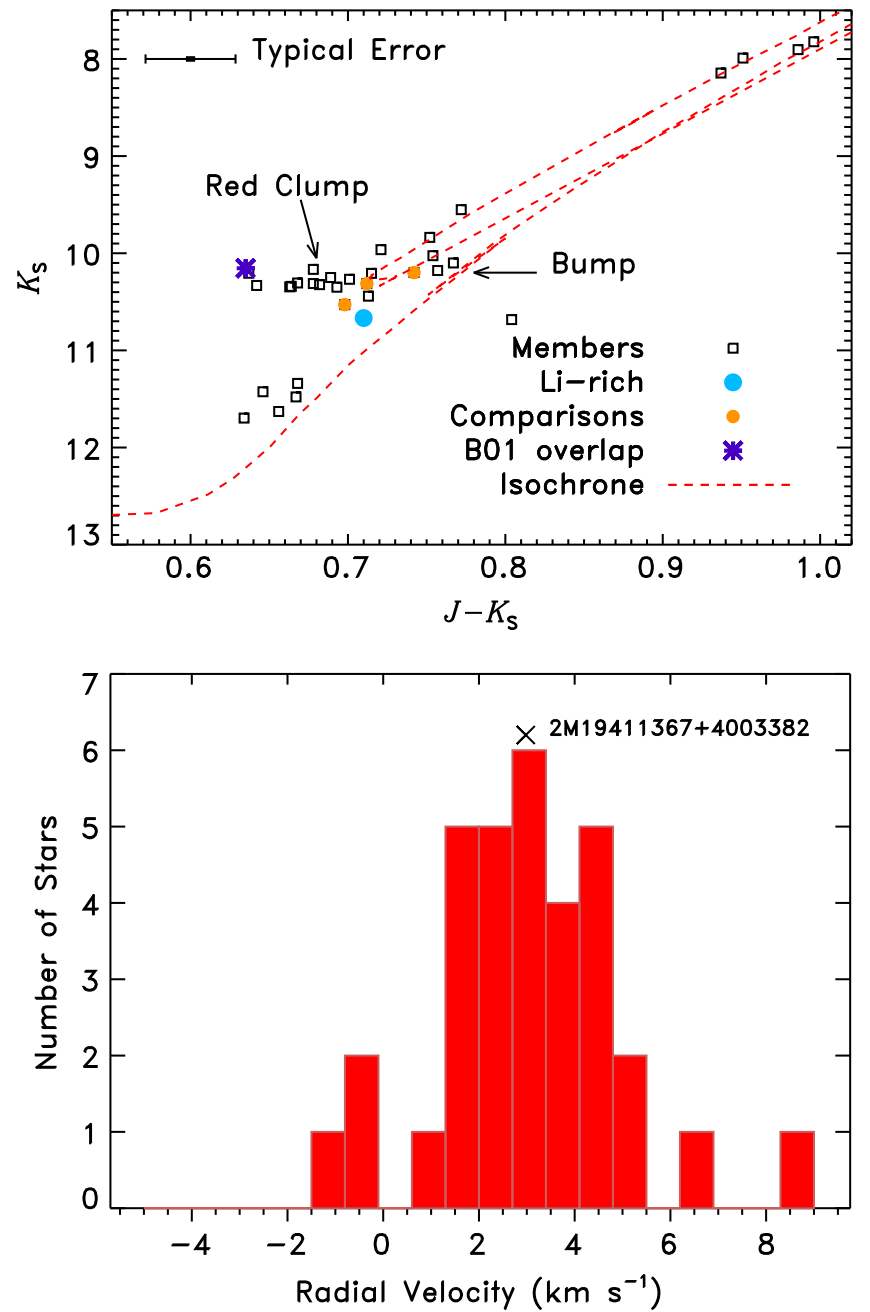

Figure 1. Top: 2MASS color-magnitude diagram of NGC 6819 member stars (black squares), 2M19411367+4003382 (large blue circle), the three comparison stars (small orange circles), and the star overlapping the optical study of Bragaglia et al. (2001, purple asterisk). Bottom: radial velocity histogram of NGC 6819 members. The $\times$ marks the RV of the Li-rich star.

The currently favored model for $2 \mathrm{M} 19411367+4003382$ is the mixing model of D12, which can explain Li-rich giants below the luminosity bump. In addition to enriching the surface with $\mathrm{Li}$, the mixing alters the star's evolution, making it follow a more extended "retrograde" evolutionary path down the red giant branch (RGB) compared to normal luminosity bump evolution. In the discovery paper, AT13 argued that $2 \mathrm{M} 19411367+4003382$ 's $\mathrm{Li}$ abundance and position on the RGB was most consistent with this model, but they also noted that a crucial test of this hypothesis is the measurement of ${ }^{12} \mathrm{C} /{ }^{13} \mathrm{C}$ to confirm whether the extra mixing has indeed occurred.

What makes 2 M19411367+4003382 such a powerful test of Li-regeneration hypotheses is its apparent membership in an open cluster, which allows a direct comparison of its abundances to other stars of nearly identical evolution. However, its membership is not conclusively established. Its radial velocity $(\mathrm{RV})$, original proper motion measurement (Sanders 1972), color, and magnitude (e.g. AT13) support membership (see Figure 1), but its asteroseismic properties (Stello et al. 2011) and more recently measured proper motion (Platais et al. 2013) support non-membership. Both of these non-membership criteria have caveats. Platais et al. (2013) reported that stars with high RV membership probabilities but low proper motion membership probabilities tend to have larger proper motion errors than stars of similar brightness. This suggests that there may have been confusion in the star's identification on the photographic plates. The asteroseismic properties of the Li-rich star suggest a lower $\log g$ and mass than expected for cluster membership, but they yield a luminosity that places the star near the distance of the cluster. (These caveats are discussed in more detail in Section 7.2.)

In this paper, we use high-resolution, infrared spectra to address questions on the Li-rich star's cluster membership and evolutionary status. We select three similarly evolved comparison RGs in NGC 6819 (Section 2) and measure abundances of ten elements in the Li-rich star and comparison stars (Section 3). We measure a spectroscopic $\log g$, and find that it is consistent with the asteroseismic $\log g$ (Section 4). The spectra also reveal that the Li-rich star is rotating slightly more rapidly than the other RGs (Section 5). We combine our data and literature resources to consider possible contamination from companions or unrelated background sources (Section 6). Finally, we explore the implications of these results under the two possible cases of cluster membership (Section 7) and summarize our findings (Section 8).

\section{SELECTION OF COMPARISON STARS}

The stars in this paper were observed with the Sloan Digital Sky Survey (SDSS) telescope (Gunn et al. 2006) and the Apache Point Observatory Galactic Evolution Experiment (APOGEE) instrument (Allende Prieto et al. 2008; Majewski 2012) as part of the open cluster calibration sample for the APOGEE survey (Zasowski et al. 2013; Meszaros et al. 2013). We analyzed the Li-rich star and three similarly evolved RGs in NGC 6819, using an infrared CMD to select stars most similar to the Li-rich star, as illustrated in Figure 1. All of the NGC 6819 member stars observed by APOGEE are shown in Figure 1 together with a $2.25 \mathrm{Gyr}, Z=0.023$ isochrone (Marigo et al. 2008) shifted by $(m-M)_{0}=11.85$ (Basu et al. 2011), and $E\left(J-K_{s}\right)=0.07$ (from $E(B-V$ ) $=0.14 \mathrm{mag}$, Bragaglia et al. 2001). The Li-rich star sits below the RC and blueward of the luminosity bump of the isochrone. The same is true of the most similar control star (2M19404965 $+4014313)$. Another comparison star is near the red edge of the RC (2M19412222+4016442), and the remaining comparison star (M19412176+4012111) overlaps the luminosity bump within the uncertainties. The 2MASS and KIC identifiers of these fours stars are listed in Table 1. Additionally, 2M19411476+4011008 was selected for analysis solely because it has previously reported spectroscopic abundances and $\log g$ derived from optical spectra (Bragaglia et al. 2001, hereafter B01). This star, identified as 978 in B01, is hotter than the Li-rich star by $\sim 200 \mathrm{~K}$ and sits at the blue edge of the $\mathrm{RC}$. Because this star is much more evolved, it is not a good comparison star to the Li-rich star, and we provide results for this star separately in Table 2.

From the 2MASS colors and isochrones, we obtain the temperature, gravity and luminosity listed in the top section of Table 1. $T_{\text {eff }}$ is the average of two $\left(J-K_{s}\right)_{0}$ to $T_{\text {eff }}$ calibrations (Bessell et al. 1998 and González Hernández and Bonifacio 2009). The uncertainty in $\left(J-K_{S}\right)_{0}$ from the 2MASS photometry is typically $0.028 \mathrm{mag}$, leading to a temperature uncertainty of $\sim 80 \mathrm{~K}$ in each calibration. The Bessell et al. 
Table 1

Measured Properties of NGC 6819 Red Giants

\begin{tabular}{|c|c|c|c|c|}
\hline Property & Li-rich & $\begin{array}{l}\text { Comparison \#1 } \\
\text { (Most Similar) }\end{array}$ & $\begin{array}{c}\text { Comparison \#2 } \\
\text { (Near RC) }\end{array}$ & $\begin{array}{c}\text { Comparison \#3 } \\
\text { (Near Bump) }\end{array}$ \\
\hline 2MASS & $2 \mathrm{M} 19411367+4003382$ & $2 \mathrm{M} 19404965+4014313$ & $2 \mathrm{M} 19412222+4016442$ & $2 \mathrm{M} 19412176+4012111$ \\
\hline KIC & 4937011 & 5111940 & 5112744 & 5112734 \\
\hline$T_{\text {eff }}($ ASPCAP $)$ & 4670 & 4687 & 4645 & 4632 \\
\hline $\log L / L_{\odot}$ & 1.52 & 1.59 & 1.67 & 1.69 \\
\hline $\log g^{\mathrm{a}}$ & 2.8 & 2.7 & 2.6 & 2.6 \\
\hline$A(\mathrm{Li})(\mathrm{dex})^{\mathrm{b}}$ & 2.3 & $\lesssim 0.7$ & $\lesssim 0.7$ & $\lesssim 0.7$ \\
\hline \multicolumn{5}{|c|}{ APOGEE-derived } \\
\hline $\log g$ & 2.35 & 2.53 & 2.51 & 2.62 \\
\hline$A\left({ }^{12} \mathrm{C}\right)$ & $8.48 \pm 0.06$ & $8.34 \pm 0.05$ & $8.39 \pm 0.05$ & $8.38 \pm 0.02$ \\
\hline$A\left({ }^{14} \mathrm{~N}\right)$ & $8.11 \pm 0.05$ & $8.15 \pm 0.02$ & $8.13 \pm 0.02$ & $8.16 \pm 0.03$ \\
\hline$A(\mathrm{Mg})$ & $7.44 \pm 0.11$ & $7.48 \pm 0.11$ & $7.45 \pm 0.13$ & $7.47 \pm 0.12$ \\
\hline$A(\mathrm{Al})$ & $6.32 \pm 0.03$ & $6.47 \pm 0.05$ & $6.45 \pm 0.04$ & $6.46 \pm 0.04$ \\
\hline$A(\mathrm{Si})$ & $7.50 \pm 0.06$ & $7.51 \pm 0.06$ & $7.51 \pm 0.08$ & $7.53 \pm 0.09$ \\
\hline$A(\mathrm{Ti})$ & $4.93 \pm 0.17$ & $5.02 \pm 0.07$ & $5.02 \pm 0.04$ & $5.03 \pm 0.12$ \\
\hline$A(\mathrm{Fe})$ & $7.45 \pm 0.12$ & $7.52 \pm 0.04$ & $7.48 \pm 0.12$ & $7.48 \pm 0.08$ \\
\hline$A(\mathrm{Ni})$ & $6.22 \pm 0.07$ & $6.19 \pm 0.06$ & $6.22 \pm 0.08$ & $6.22 \pm 0.05$ \\
\hline$v \sin i\left(\mathrm{~km} \mathrm{~s}^{-1}\right)$ & $8.5 \pm 1.1$ & $6.6 \pm 1.6$ & $4.4 \pm 1.5$ & $5.8 \pm 1.8$ \\
\hline \multicolumn{5}{|c|}{ APOKASC } \\
\hline$\nu_{\max }(\mu \mathrm{Hz})$ & 29.51 & 52.33 & 43.69 & 40.11 \\
\hline$\Delta \nu(\mu \mathrm{Hz})$ & 4.15 & 5.16 & 4.39 & 4.12 \\
\hline $\log g$ & 2.37 & 2.62 & 2.54 & 2.50 \\
\hline
\end{tabular}

${ }^{a} \log g$ from the isochrone and used to derive abundances.

b Measurement for 2M19411367+4003382 from AT13; their Figure 3 provides estimates for comparison stars.

(1998) calibration uses the derived color indices for ATLAS9 overshoot models (their Table 2) and gives slightly cooler temperatures than the González Hernández and Bonifacio (2009) calibrations by $40-60 \mathrm{~K}$. This systematic difference in temperature is well within the $1 \sigma$ uncertainties of each calibration. The resulting temperatures are also in good agreement (all within $55 \mathrm{~K}$ ) with those derived by the APOGEE Stellar Parameters and Chemical Abundance Pipeline (ASPCAP, A. E. Pérez García et al., 2015 in preparation). We can also tie this temperature scale to an independent spectroscopic analysis. The comparison star overlapping the B01 study has $T_{\text {eff }}=4855 \mathrm{~K}$ derived from optical spectra compared to $T_{\mathrm{ASPCAP}}=4867 \mathrm{~K}$, and our $T_{\mathrm{eff}}=4920 \mathrm{~K}$.

Assuming a stellar mass of $1.7 M_{\odot}$ from the isochrone, we derive the surface gravities in the top section of Table 1 . These are the $\log g$ 's used to derive abundances. For all of the models, we adopted a microturbulence $\xi$ of $1.5 \mathrm{~km} \mathrm{~s}^{-1}$, following the prescription adopted by ASPCAP $(\xi=2.24-0.3 \log g)^{24}$ for $\log g=2.6$ dex.

\footnotetext{
${ }^{24}$ See http://sdss3.org/dr10/irspec/aspcap.php\#aspcap
}

\section{ABUNDANCES FROM APOGEE SPECTRA}

The spectrum for each star has been processed with the APOGEE pipeline (Nidever et al., 2015). The observed data used here are the continuum-normalized "aspcapStar" spectra, with the exception of the wavelength region near the Ti II line, which falls near one of the detector gaps and is masked out in the "aspcapStar" spectra. This spectral region comes from the "apStar" spectra, which we continuum normalize. All spectra are described and are available in the tenth data release (DR10, Ahn et al. 2014) of the SDSS-III (Eisenstein et al. 2011). ${ }^{25}$

Abundances are measured via spectrum synthesis using MOOG (Sneden 1973) ${ }^{26}$ and the APOGEE linelist (M. Shetrone et. al., 2015 in preparation), focusing on small subsets of atomic and molecular features identified for each element by Smith et al. (2013, hereafter S13). All of the wavelengths in this paper refer to air wavelengths. A spectrum of a $\sim 20 \AA$ region around each feature under consideration was generated using MOOG, smoothed by a Gaussian to match the broadening of the observed spectra. Small adjustments were

\footnotetext{
$25 \mathrm{https} / / / \mathrm{sdss} 3.0 \mathrm{rg} / \mathrm{dr} 10 /$

${ }^{26}$ MOOG is available at http://as.utexas.edu/ chris/moog.html.
} 
Table 2

Properties of 2M19411476+4011008 Compared to Literature

\begin{tabular}{lccc}
\hline \hline Property & This Work & B01 & $\Delta($ This Work - B01) \\
\hline$T_{\text {eff }}$ & 4920 & 4855 & +65 \\
$\log L / L_{\odot}$ & 1.79 & $\ldots$ & $\ldots$ \\
$\log g^{\mathrm{a}}$ & 2.6 & 2.60 & +0.00 \\
$\log g^{\mathrm{b}}$ & 2.58 & 2.60 & -0.02 \\
$\xi\left(\mathrm{km} \mathrm{s}^{-1}\right)$ & 1.5 & 1.26 & +0.24 \\
$A\left({ }^{12} \mathrm{C}\right)$ & $8.32 \pm 0.08$ & $\ldots$ & $\ldots$ \\
$A\left({ }^{14} \mathrm{~N}\right)$ & $8.21 \pm 0.03$ & $\ldots$ & $\ldots$ \\
$A\left({ }^{16} \mathrm{O}\right)$ & $8.74 \pm 0.16$ & $\ldots$ & $\ldots$ \\
$\mathrm{C} / \mathrm{N}$ & 1.3 & $\ldots$ & $\ldots$ \\
${ }^{12} \mathrm{C} /{ }^{13} \mathrm{C}$ & $>20$ & $\ldots$ & -0.50 \\
$A(\mathrm{Na})$ & $6.46 \pm 0.07$ & 6.96 & -0.09 \\
$A(\mathrm{Mg})$ & $7.56 \pm 0.09$ & 7.65 & +0.12 \\
$A(\mathrm{Al})$ & $6.56 \pm 0.02$ & 6.44 & -0.22 \\
$A(\mathrm{Si})$ & $7.60 \pm 0.03$ & $7.82 \pm 0.14$ & -0.18 \\
$A(\mathrm{Ti})$ & $5.04 \pm 0.08$ & $5.22 \pm 0.16$ & -0.02 \\
$A(\mathrm{Fe})$ & $7.58 \pm 0.06$ & $7.60 \pm 0.12$ & -0.09 \\
$A(\mathrm{Ni})$ & $6.28 \pm 0.06$ & $6.37 \pm 0.14$ & $\ldots$ \\
$v \sin i\left(\mathrm{~km} \mathrm{~s}^{-1}\right)$ & $3.4 \pm 1.3$ & $\ldots$ & \\
\hline
\end{tabular}

${ }^{a} \log g$ from the isochrone.

${ }^{\mathrm{b}} \log g$ from ionization balance.

made to the continuum level and velocity shift of the observed data to best match the models.

\subsection{C, N, O, and ${ }^{12} \mathrm{Cl}^{13} \mathrm{C}$}

The abundances of $\mathrm{C}, \mathrm{N}$, and $\mathrm{O}$ must be found iteratively because they are inter-related by molecular equilibrium conditions. We first measure $\mathrm{C}$ abundances from $\mathrm{CO}$ lines, then fix the $\mathrm{C}$ abundances to measure $\mathrm{O}$ using $\mathrm{OH}$ lines. These steps are iterated until convergence is reached. The $\mathrm{N}$ abundances can then be derived from $\mathrm{CN}$ lines. The lines used in this analysis are from Table 4 of S13, excluding the ${ }^{12} \mathrm{C}^{16} \mathrm{O}(4-1)$ V-R lines, the ${ }^{16} \mathrm{OH}(3-1) \mathrm{P}_{2} 9.5$, and the ${ }^{12} \mathrm{C}^{14} \mathrm{~N}$ $(0-1) \mathrm{R} 168.5$ lines. These abundances and the $\mathrm{C} / \mathrm{N}$ values are listed in the middle section of Table 1 for the Li-rich star and comparison stars and in Table 2 for 2M19411476+4011008. The uncertainties are the standard deviations of the individual measurements, and we address the uncertainties propagated from the stellar parameter uncertainties in the next section. The three comparison stars all have nearly identical abundances, but the Li-rich star appears to have somewhat higher O. The low $\mathrm{C} / \mathrm{N}$ confirms that standard FDU has completed and Li dilution should have occurred in all of the stars.

To measure ${ }^{12} \mathrm{C} /^{13} \mathrm{C}$, we only used the two ${ }^{13} \mathrm{C}^{14} \mathrm{~N}$ lines in $\mathrm{S} 13$ because the ${ }^{13} \mathrm{C}^{16} \mathrm{O}$ features are weak and contaminated with night sky emission lines. The ${ }^{13} \mathrm{C}^{14} \mathrm{~N}$ lines are also quite weak in all of the stars analyzed, which indicates high ${ }^{12} \mathrm{C} /{ }^{13} \mathrm{C}$. We illustrate the measurement of ${ }^{12} \mathrm{C} /{ }^{13} \mathrm{C}$ from the Li-rich star's spectrum in Figure 2. Low ${ }^{12} \mathrm{C} /{ }^{13} \mathrm{C}(\lesssim 15)$ are clearly excluded by the weakness of the ${ }^{13} \mathrm{C}^{14} \mathrm{~N}$. The fits favor ${ }^{12} \mathrm{C} /{ }^{13} \mathrm{C}$ $\sim 20-30$, consistent with normal levels of isotopic ${ }^{13} \mathrm{C}$ enrichment in the stellar envelope following FDU. The constraints against high ${ }^{12} \mathrm{C} /{ }^{13} \mathrm{C}$, i.e., $>30$, are obviously weaker than those against low ${ }^{12} \mathrm{C} /{ }^{13} \mathrm{C}$, but our $\mathrm{C} / \mathrm{N}$ measurement gives us confidence that FDU has occurred.

In Figure 3, we compare our mixing indicators $(\mathrm{C} / \mathrm{N}$ and ${ }^{12} \mathrm{C} /{ }^{13} \mathrm{C}$ ) to RGs in other open clusters. We plot the mixing indicators as a function of the clusters' turn-off mass $\left(M_{\mathrm{TO}}\right)$. The literature values come from a series of papers focusing on the abundances of open cluster stars (Mikolaitis et al. 2011a, 2011b, 2012). The first paper compiles even earlier studies from Gilroy (1989), Luck (1994), Tautvaišiene et al. (2000), Tautvaišiené et al. (2005), Smiljanic et al. (2009), and Mikolaitis et al. (2010). Figure 3 demonstrates that the stars in this paper generally fit within the trends defined by the other clusters. The exception is $\mathrm{C} / \mathrm{N}$ for the Li-rich star, which is somewhat high for a star of its presumed mass. Oxygen is not processed in stars of this mass range, so we expect $[\mathrm{O} / \mathrm{Fe}]$ to remain near zero. We find that the comparison stars have $[\mathrm{O} / \mathrm{Fe}]$ between -0.03 and 0.11 , which is consistent with our expectation given the uncertainties of $\sim 0.1$ dex. However, the Li-rich star again stands out. It has $[\mathrm{O} / \mathrm{Fe}]=0.3$ \pm 0.16 dex, suggestive of an unusual enhancement in O. Extra deep mixing would be expected to lower the [O/Fe], e.g., as in the case of IRS 7 (Carr \& Sellgren 2000).

\section{2. $\mathrm{Na}, \mathrm{Mg}, \mathrm{Al}, \mathrm{Si}, \mathrm{Ti}, \mathrm{Fe}$, and $\mathrm{Ni}$}

The full line lists in Table 5 of S13 were used for Fe I, Mg I, and Al I. Subsets of the S13 list were used for Si I (15376.8, $15960.1,16060.0,16094.8,16215.7,16680.8,16282.2 \AA), \mathrm{Ni}$ I $(15632.7, \quad 16584.4, \quad 16589.3, \quad 16673.7 \AA)$, and $\mathrm{Ti}_{\mathrm{I}}$ $(15543.756 \AA$ and $15602.842 \AA$ ). Sodium abundances were measured from two $\mathrm{Na}$ I lines (16373.9 and $16388.9 \AA)$ identified in Cunha et al. (2015). We fixed the C, N, and O abundances found in Section 3.1 for these analyses. The abundance results are given in Table 1 for the main sample, and the uncertainties are again the standard deviation of the measurements.

In Table 2 we compare our results for 2M19411476 +4011008 to the abundances derived from optical data by B01. Columns two and three list the abundances and standard deviations $(\sigma)$ measured here and in B01, respectively. When only one line for a given element was measured by B01, we do not list $\sigma$. The last column shows the differences between the abundances derived here and those derived by B01. Most of our abundances agree within the quoted uncertainties. The exceptions are $\mathrm{Si}$ and $\mathrm{Na}$. For both of these elements, we find lower abundances than B01. Compared to Comparisons 1-3, we generally measure slightly larger abundances for 2M19411476+4011008, although only Al disagrees with the other cluster stars outside the uncertainties.

In Figure 4, we present our elemental abundance measurements (solar values from Asplund et al. 2009) of the Li-rich star and the three comparison stars. Since all of these stars have similar evolutionary stages and stellar parameters, we would expect them to all have similar abundances. (We exclude the star overlapping B01 from this plot because it is at a much more evolved evolutionary stage.) The abundances of the Lirich star are generally in good agreement with the Li-normal comparison stars, with the exceptions being $\mathrm{O}, \mathrm{Na}$, and $\mathrm{Al}$. The enhanced $A(\mathrm{O})$ was already mentioned in the previous section. The $A(\mathrm{Al})$ of the Li-rich star is $\sim 0.14$ dex lower than the three most similar comparison stars-well outside the $0.05 \mathrm{dex}$ uncertainties. $A(\mathrm{Na})$ is also quite low in the Li-rich star, though the uncertainties are large. However, this large uncertainty means that the two $\mathrm{Na}$ lines disagree. If we directly compare each line in the Li-rich star to the most similar control star, we find that both $\mathrm{Na}$ lines are significantly weaker in the Li-rich star, pointing to a true deficiency in Na. Thus, both $A(\mathrm{Al})$ and 

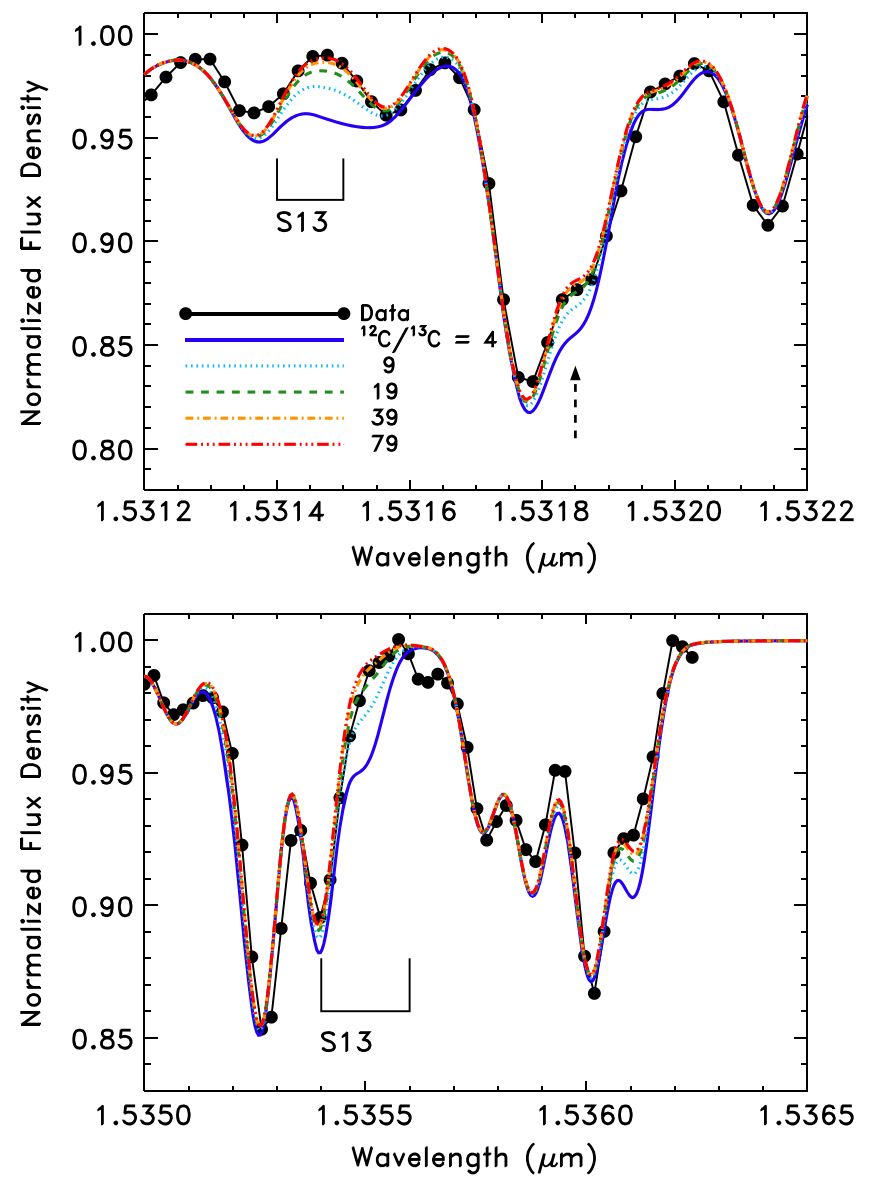

Figure 2. Measurement of ${ }^{12} \mathrm{C} /{ }^{13} \mathrm{C}$ from ${ }^{13} \mathrm{C}^{14} \mathrm{~N}$ lines for the Li-rich star (circles). The wavelength intervals of S13 are marked. The synthetic spectra (colored lines) span a range of ${ }^{12} \mathrm{C} /{ }^{13} \mathrm{C}$. Top: Low ${ }^{12} \mathrm{C} /{ }^{13} \mathrm{C}$ are ruled out in the $\mathrm{S} 13$ wavelength interval, but continuum level uncertainties make it difficult to place a stringent lower limit. A neighboring region (arrow) suggests ${ }^{12} \mathrm{C} /{ }^{13} \mathrm{C} \sim$ 20. Bottom: The wavelength interval of $\mathrm{S} 13$ favors ${ }^{12} \mathrm{C} /{ }^{13} \mathrm{C} \sim 30$.

$A(\mathrm{Na})$ may be low for the Li-rich star. If the reverse were true, the anomalous abundances could be explained by extra-mixing, which enhances the surface abundance of $\mathrm{Li}, \mathrm{Na}$, and $\mathrm{Al}$.

\subsection{Abundance Sensitivities}

To estimate the sensitivity of the abundance measurements to uncertainties in the stellar parameters used to select a model atmosphere for each star, we selected a fiducial model of $T_{\text {eff }}=4700 \mathrm{~K}, \log g=2.7 \mathrm{dex},[\mathrm{M} / \mathrm{H}]=0.0$, and $\xi=1.5$ $\mathrm{km} \mathrm{s}^{-1}$ and tested how the output abundances change for perturbations of $\Delta T_{\text {eff }}= \pm 80 \mathrm{~K}, \quad \Delta \log g= \pm 0.2$ dex, $\Delta[\mathrm{M} / \mathrm{H}]= \pm 0.1$ dex, and $\Delta \xi= \pm 0.2 \mathrm{~km} \mathrm{~s}^{-1}$. This was accomplished by adopting mean abundances of each element from our stellar sample, calculating the equivalent width needed to produce that abundance with the fiducial model for each line, and fixing the equivalent width to see how the output abundance changed with changes in the model atmosphere. The changes for all lines of a given element are then averaged and presented in Table 3. Each entry in the table gives two numbers. The first number is the change in abundance when increasing the stellar parameter by the specified amount, and the second number is the change in abundance when the stellar parameter is decreased. For the sensitivities of $\mathrm{C}, \mathrm{N}$, and $\mathrm{O}$, we adopted a simplified approach by using the line sensitivities of

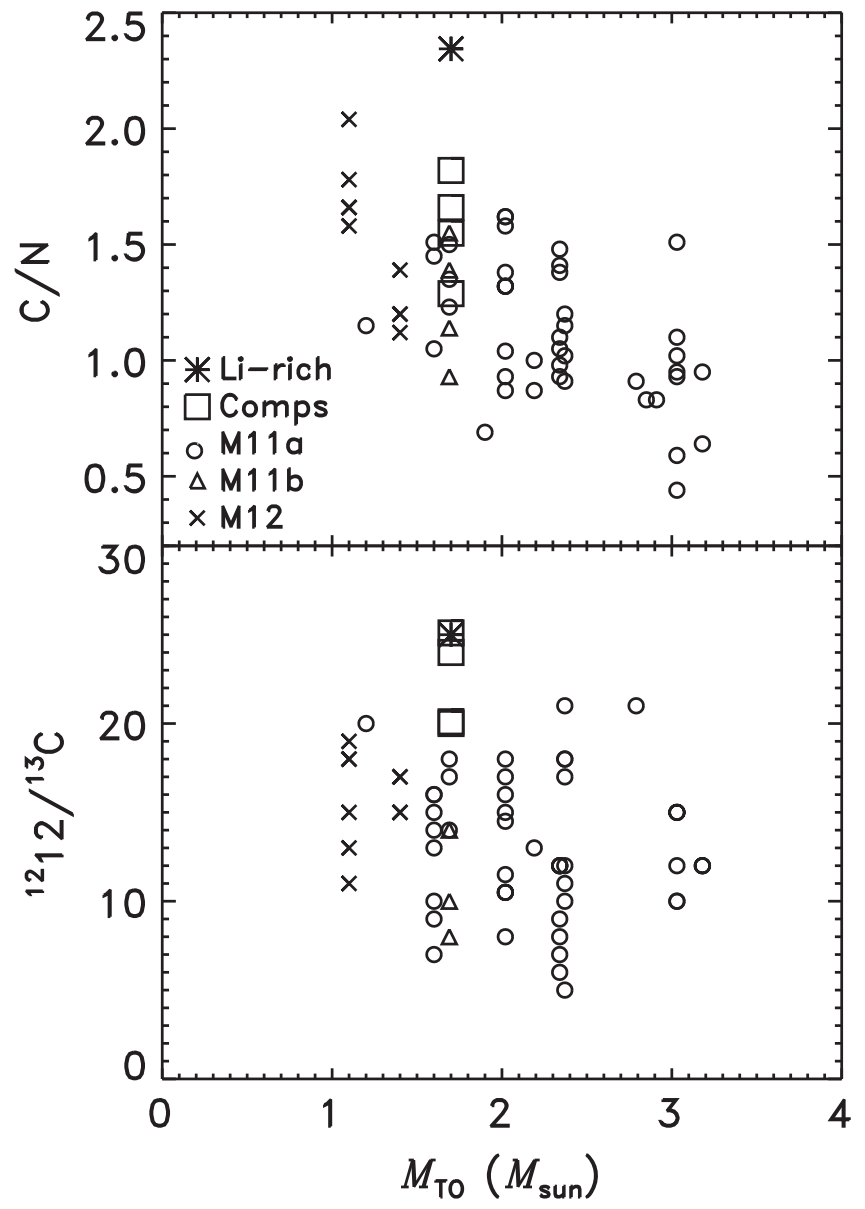

Figure 3. Relationship between cluster turn-off mass and $\mathrm{C} / \mathrm{N}$ (top) and ${ }^{12} \mathrm{C} /{ }^{13} \mathrm{C}$ (bottom). The Li-rich star (asterisks) and comparison stars (squares) are compared to other open clusters in the literature (Mikolaitis et al. 2011a, circles; 2011b, triangles; 2012, x).

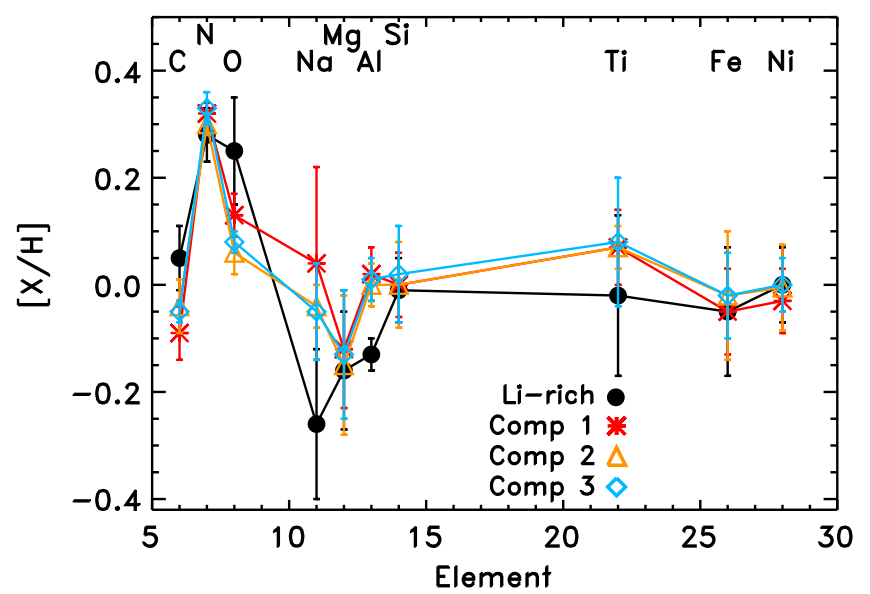

Figure 4. Elemental abundances normalized to solar (Asplund et al. 2009) of the Li-rich star and three comparison stars.

the three molecules present in our line list as a proxy for either $\mathrm{C}, \mathrm{N}$, or $\mathrm{O}$. The molecules in Table 3 have their associated elements given in parentheses. 
Table 3

Abundance Sensitivities for the Fiducial Model

\begin{tabular}{|c|c|c|c|c|}
\hline Species & $\begin{array}{c}\Delta A / \Delta T_{\text {eff }} \\
\quad \pm 80 \mathrm{~K}\end{array}$ & $\begin{array}{c}\Delta A / \Delta \log g \\
\pm 0.2 \operatorname{dex}\end{array}$ & $\begin{array}{c}\Delta A / \Delta[M / H] \\
\quad \pm 0.1 \mathrm{dex}\end{array}$ & $\begin{aligned} & \Delta A / \Delta \xi \\
\pm & 0.2 \mathrm{~km} \mathrm{~s}^{-1}\end{aligned}$ \\
\hline $\mathrm{CO}(\mathrm{C})$ & $+0.049 /-0.055$ & $+0.065 /-0.063$ & $+0.029 /-0.042$ & $-0.003 /+0.002$ \\
\hline $\mathrm{CN}(\mathrm{N})$ & $+0.037 /-0.021$ & $+0.053 /-0.035$ & $+0.017 /-0.033$ & $-0.014 /+0.015$ \\
\hline $\mathrm{OH}(\mathrm{O})$ & $+0.101 /-0.097$ & $-0.017 /+0.040$ & $+0.087 /-0.107$ & $-0.002 /+0.002$ \\
\hline $\mathrm{Na} \mathrm{I}$ & $+0.042 /-0.042$ & $-0.007 /+0.027$ & $-0.002 /-0.016$ & $-0.002 /+0.003$ \\
\hline Mg I & $+0.042 /-0.038$ & $-0.041 /+0.064$ & $+0.018 /-0.036$ & $-0.012 /+0.013$ \\
\hline $\mathrm{Al}$ I & $+0.070 /-0.071$ & $-0.074 /+0.102$ & $+0.020 /-0.044$ & $-0.033 /+0.030$ \\
\hline Si I & $+0.021 /-0.015$ & $-0.044 /+0.062$ & $+0.040 /-0.053$ & $-0.024 /+0.023$ \\
\hline Ti I & $+0.111 /-0.109$ & $-0.001 /+0.016$ & $-0.005 /-0.012$ & $-0.045 /+0.054$ \\
\hline Ti II & $-0.019 /+0.022$ & $+0.078 /-0.092$ & $+0.035 /-0.036$ & $-0.035 /+0.038$ \\
\hline $\mathrm{Fe}_{\mathrm{I}}$ & $+0.050 /-0.045$ & $-0.032 /+0.046$ & $+0.026 /-0.042$ & $-0.040 /+0.043$ \\
\hline $\mathrm{Ni}$ I & $+0.019 /-0.014$ & $+0.006 /+0.008$ & $+0.020 /-0.033$ & $-0.017 /+0.018$ \\
\hline
\end{tabular}

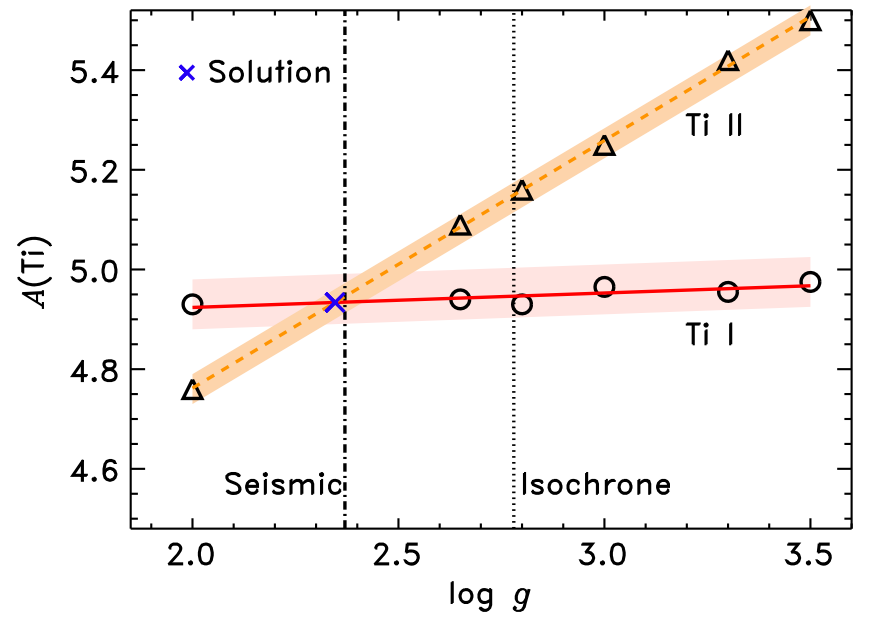

Figure 5. Ionization equilibrium plot for the Li-rich star. The shaded regions around $\mathrm{Ti}$ I (circles) and $\mathrm{Ti}$ II (triangles) show individual line uncertainty measurements of \pm 0.05 and \pm 0.03 dex, respectively. The intersection of these relations $(x)$ yields the $\log g$. Vertical lines mark the expected $\log g$ from isochrones (dotted line) and asteroseismology (dashed-dotted line).

\section{SURFACE GRAVITY}

\subsection{Spectroscopic $\log g$}

For elements with low ionization potential that are predominantly ionized in the photospheres of the stars of interest, the strengths of lines of singly ionized atomic lines are sensitive to the $\log g$ of the star, while neutral atomic lines are not. When lines of both species of an element are available, they can be used to measure $\log g$. In the APOGEE spectral range, the Ti II line at $15873.834 \AA$ is the sole known ionized line. Wood et al. (2014) reported an experimentally measured $\log g f=-1.90 \pm 0.08$ for this line, while the APOGEE team adopted an astrophysical $\log g f=-2.06$ from fitting spectra of the Sun and Arcturus. The latter is adopted here. We measured the strength of this $\mathrm{Ti}$ II feature and the $\mathrm{Ti}$ I lines for our five stars while varying the $\log g$ of the atmosphere models from 2.0 to 3.5 dex, as illustrated in Figure 5 for the Li-rich star. Our spectroscopic $\log g$ is the one for which $A(\mathrm{Ti})$ of $\mathrm{Ti}$ I and $\mathrm{Ti}$ II are equal. These $\log g$ measurements are listed in the second section of Table 1, and they generally agree well with those predicted from the cluster isochrone. The exception is the Lirich star. We find $\log g=2.35$ dex compared to the isochrone value of $2.8 \mathrm{dex}$; however, this is a good match to the $\log g$ derived from asteroseismology (Section 4.2).
To check the impact of having a single ionized line with an uncertain oscillator strength, we check for a systematic offset in $\log g$ using the star that overlaps the B01 study. They measured $\log g$ with the ionization balance of $\mathrm{Fe}$ and find $\log g=2.6$ dex. This agrees with both our ionization balance $(2.58 \mathrm{dex})$ and isochrone $(2.6 \mathrm{dex})$ results.

\subsection{Asteroseismic $\log g$}

An additional gravity measure comes from the asteroseismic $\nu_{\max }$ in the APOKASC catalog (Pinsonneault et al. 2014), using the standard scaling relationship where $g \propto \nu_{\max } \sqrt{T}_{\text {eff }}$ (Kjeldsen $\&$ Bedding 1995). These gravities are listed in the bottom section of Table 1. In all cases, the asteroseismic gravities agree well with our spectroscopic $\log g$. In turn, the spectroscopic $\log g$ matches well with the isochrone measurement for all but the Li-rich star. The Li-rich star has a spectroscopic $\log g$ that is in good agreement with the asteroseismic value, but it is 0.4 dex lower than what is expected from isochrones. Unfortunately, 2M19411476+4011008 does not have a $\nu_{\max }$ measurement so we do not have a direct comparison between the asteroseismic $\log g$ and $\log g$ derived from ionization balance of Fe lines.

\section{ROTATIONAL VELOCITY}

While measuring abundances, we required a slightly larger broadening of the synthetic spectra to fit the lines of the Li-rich spectrum compared to the other spectra. We acquired the rotational velocities of the stars in this study from the pipeline being developed for APOGEE (D. Bizyaev etal., 2015 in preparation). Briefly, the spectra are cross-correlated with synthetic templates that have been convolved with a rotational broadening profile for a range of $v \sin i$. The results of this analysis are listed in Table 1. As expected, the Li-rich star has the largest $v \sin i$, and the comparison stars have $v \sin i$ near the detection limit for APOGEE's resolution. The clear measurement of $v \sin i=8.5 \mathrm{~km} \mathrm{~s}^{-1}$ points to unusually fast rotation in the Li-rich star. Such a high $v \sin i$ for RGs is rare. In a recent survey for fast rotators among open cluster RGs, Carlberg (2014) found only one RG out of $\sim 270$ cluster members that rotate as rapidly as the Li-rich star.

\section{BINARY COMPANIONS OR OTHER BLENDS}

We performed multiple checks of whether our spectroscopic analysis is affected by either a companion to the Li-rich star or an unrelated fore/background object. First, we fit model 
spectral energy distributions (SED) to photometry spanning optical (Hole et al. 2009), near-IR (2MASS, Skrutskie et al. 2006), and mid-IR (WISE, Wright et al. 2010) wavelengths, allowing the temperature of the models and visual extinction to be free parameters. The best results are obtained for a bare photosphere with a hotter temperature $\left(T_{\text {eff }}\right.$ $\sim 5000 \mathrm{~K})$ than that derived from our $\left(J-K_{s}\right)_{0}$ calibration and a larger line of sight extinction. This solution implies that the Li-rich star is a more distant star affected by additional extinction. However, the analysis favors the largest extinction we allowed in the fit, $A_{V}=0.665$, which is slightly higher than $A_{V}=0.623$, the extinction in the Schlegel et al. (1998) map at this position. Additionally, the same SED analysis of the most similar comparison star also favors a hotter, more reddened solution. Therefore, we conclude that the temperature difference may be systematic and cannot discriminate the Li-rich stars's cluster membership. We also find no evidence for an IR excess out to $10 \mu \mathrm{m}$.

APOGEE made three unique observations of the Li-rich star, roughly evenly spaced over the course of a month. The RV is constant within the $0.1-0.2 \mathrm{~km} \mathrm{~s}^{-1}$ uncertainties of the three observations, and the Li-rich star's RV matches that of the cluster, as illustrated in Figure 1. Admittedly, one month is a short time span to look for RV variations, but the literature contains additional RV measurements for this star spanning at least 20 years. The optical spectrum taken by AT13 was taken between 2010 and 2012 and also had an RV consistent with cluster membership. Hole et al. (2009) define the star as a single, cluster member based on four RV measurements going back at least to 1992. Taken together, all of these RVs suggest that $2 \mathrm{M} 19411367+4003382$ is a RV stable cluster member.

We also leveraged the individual visit spectra to search for evidence of a secondary spectrum by cross-correlating each visit spectrum with a library of template spectra. All three give best fits to templates of $T_{\text {eff }} \sim 5000 \mathrm{~K}$. The shape of the crosscorrelations peaks does not change between visits, and no secondary peaks are found, ruling out bright companions of similar spectral type. A second round of cross-correlations used the TODCOR algorithm (Zucker \& Mazeh 1994) to generate a two-dimensional cross-correlation using a wide range of possible secondary spectral types: no significant secondary peaks were identified. We conclude that the spectral absorption features are truly arising in the RG's atmosphere and are not significantly contaminated by flux from any binary companion or unassociated blend.

\section{INTERPRETATION OF THE Li-RICH STAR}

Our analysis of the Li-rich star has confirmed that it is evolved-its low $\log g$ and non-solar C/N are consistent with the picture of a RG that has undergone FDU and should have depleted Li. However, given the uncertainties in the cluster membership, the Li-rich star may be a more massive, more luminous background $\mathrm{RG}$ that coincidentally shares the same $\mathrm{RV},[\mathrm{Fe} / \mathrm{H}]$, and CMD locus as the cluster. Therefore, to fully explore the implications of our measurements we provide two scenarios. The first scenario presumes that the Li-rich star is not a member of NGC 6819, and we explore how that assumption changes our derived abundances, $\log g$, etc. The second scenario explores the implications of the star being a cluster member, and we demonstrate why we favor this scenario.

\subsection{Case 1: Cluster Non-membership}

\subsubsection{Underestimated Reddening}

If the Li-rich star is not a member of the cluster, then the temperature derived from the $J-K_{s}$ is a lower limit, since the reddening may be underestimated. We can test how our analysis will change if we adopt the total line-of-sight reddening observed in the direction of 2M19411367 +4003382. If we adopt $A_{V}=0.665$ (the preferred solution of the SED fitting), then $E(B-V)=0.215$ and $E\left(J-K_{s}\right)=0.107$. Reapplying our temperature calibration yields $4840 \mathrm{~K}$. The strong temperature dependence of Ti I lines increases $A(\mathrm{Ti}$ I), while Ti II remains nearly constant. Thus, the ionization equilibrium plot (Figure 5 ) for $T_{\text {eff }}=4840 \mathrm{~K}$ would have a similar shape, but the horizontal line would shift upwards by $\sim 0.2 \mathrm{dex}$ and intersect the $A(\mathrm{Ti}$ II $)$ line at $\log g=2.81$ and $A(\mathrm{Ti})=5.14 \mathrm{dex}$. This means that if the Lirich star is in indeed a background $R G$, then its surface gravity is higher than that predicted by the asteroseismology and matches that expected from the stellar isochrones.

Since the Li-rich star has the same metallicity as the confirmed cluster stars, we can adopt solar metallicity stellar evolution tracks (Bertelli et al. 2008, 2009) to find the range of masses and ages of stars near this $T_{\text {eff }}$ and $\log g$ but that have luminosities exceeding that adopted in Table 1 under the assumption of cluster membership. We find that stars within $T_{\text {eff }}=4840 \pm 80 \mathrm{~K}, \log g=2.81 \pm 0.2 \mathrm{dex}$, and $\log L / L_{\odot}$ $\geqslant 1.52$ span a mass range of $1.8-3.0 M_{\odot}$ and an age range of 0.4-1.6 Gyr. The lowest mass stars in this range must be RC stars rather than first ascent stars in order to meet all of the selection criteria. First ascent stars are too faint. At higher masses, both first ascent stars and core He burning stars satisfy all three constraints. For the entire mass range, the stars should have completed first dilution (see, e.g., Figure 1 of Charbonnel \& Balachandran 2000) meaning the high Li abundance is still unexplained by standard stellar evolution.

Using the sensitivities calculated in Section 3.2, we can conclude that increasing the temperature by $140 \mathrm{~K}$ has the following effects: Fe changes by $+0.09, \mathrm{C}$ by $+0.09, \mathrm{~N}$ by $+0.06, \mathrm{O}$ by $+0.18, \mathrm{Na}$ by $+0.07, \mathrm{Mg}$ by $+0.07, \mathrm{Al}$ by $+0.12, \mathrm{Si}$ by +0.04 , $\mathrm{Ti}$ (from ionization balance) by +0.16 , and $\mathrm{Ni}$ by +0.03 . The new $\mathrm{C} / \mathrm{N}$ is 2.5 , which is still consistent with the completion of FDU.

\subsubsection{Internal Li Replenishment}

We can use mixing signatures to explore whether internal replenishment of $\mathrm{Li}$ is a viable mechanism for the full range of possible masses and ages of the Li-rich star. Constraints on these stellar parameters were made in the last section for the high extinction case, but we must also consider the case where the Li-rich star is affected by the same amount of extinction as the cluster stars, i.e., if there is negligible extinction between the cluster and the Li-rich star. We repeat the analysis with stellar evolution tracks for $T_{\text {eff }}=4700 \pm 80 \mathrm{~K}, \log g=$ $2.35 \pm 0.2 \mathrm{dex}$, and $\log L / L_{\odot} \geqslant 1.52$. The resulting mass range is $M=1.8-4.0 M_{\odot}$ and an age range of $0.2-1.6 \mathrm{Gyr}$. In this scenario, both first and second ascent evolutionary stages for the full mass range are consistent with the selection criteria. As a result, $1.8 M_{\odot}$ stars near the cool end of our $T_{\text {eff }}$ range $(4620 \mathrm{~K})$ are luminosity bump stars that also meet the gravity and luminosity constraint. 
In either assumed extinction scenario, the Li-rich star is an evolved giant that should have undergone convective dilution and show depleted Li. The allowed parameters of the Li-rich star includes two regimes where $\mathrm{Li}$ regeneration has been suggested to occur-the luminosity bump, and the narrow mass range of RC stars identified by Kumar et al. (2011). Thus, internal $\mathrm{Li}$ regeneration seems to be a viable explanation. However, a common feature of internal Li regeneration models is that the Li synthesis must happen deep enough in the star that whatever mechanism is ferrying the newly synthesized $\mathrm{Li}$ into the convection zone must also ferry ${ }^{13} \mathrm{C}$ and thereby lower the surface ${ }^{12} \mathrm{C} /{ }^{13} \mathrm{C}$. The absence of strong ${ }^{13} \mathrm{C}$ features excludes the deep mixing that should accompany Li-regeneration via the D12 models favored by AT13, unless we are observing the star near the beginning of the rapid $\mathrm{Li}$ enrichment stage (in which the star spends less than a few Myr with enriched Li but FDU levels of $\left.{ }^{12} \mathrm{C} /{ }^{13} \mathrm{C}\right)$.

\subsubsection{External Li Replenishment}

External replenishment models run into difficulties with the amount of material required to raise the $A(\mathrm{Li})$ to the observed value. By rearranging the equation for predicting enhanced $\mathrm{Li}$ from planet engulfment in Carlberg et al. (2012, their Equation (2)) we can write

$$
q_{\mathrm{e}}=\frac{10^{A(\mathrm{Li})_{\text {new }}}-10^{A(\mathrm{Li})_{\star}}}{10^{A(\mathrm{Li})_{\mathrm{p}}}-10^{A(\mathrm{Li})_{\text {new }}}}
$$

where $q_{\mathrm{e}}$ is the ratio of the mass of the polluting material to the mass in the stellar convective envelope, and the $A(\mathrm{Li})$ subscripts of "new," $\star$, and "p" refer to the postengulfment, the normal stellar, and pollutant abundances, respectively. Adopting $A(\mathrm{Li})_{\star}=1.49$ dex (the largest nonenriched $\mathrm{Li}), A(\mathrm{Li})_{\text {new }}=2.3 \mathrm{dex}$ (the observed abundance), and $A(\mathrm{Li})_{\mathrm{p}}=3.3$ dex (the solar nebular value), we find that $q_{\mathrm{e}}=0.09$. We used the MESA stellar evolution code (Paxton et al. 2013, version 6794) to generate limiting mass models of $M=1.7$ and $4.0 M_{\odot}$ to determine the amount of mass in the outer convection zone for stars near 4700 and $4840 \mathrm{~K}$ at different phases of the RGB evolution. In all cases, that mass was at least $1.1 M_{\odot}$. Consequently, the amount of material required to explain the enhanced $\mathrm{Li}$ in $2 \mathrm{M} 19411367+4003382$ is $0.1 M_{\odot}$, which is a stellar mass companion. Such a low mass companion would have been missed by our binary analysis. However, low mass stars are also $\mathrm{Li}$ depleted. Substellar companions retain their birth supply of $\mathrm{Li}$, but they are too low mass unless they are significantly enriched in Li over the solar nebular value.

\subsection{Case 2: Cluster Membership}

The inferences on the origin of the excess Li mentioned in the previous section are valid in the case of cluster membership, but we place further constraints on the unusual nature of the Li-rich star if it is truly a cluster member. The cluster membership is supported from a number of lines of evidence. Its RV is consistent with membership, and its optical and infrared magnitudes coincide with the cluster's CMD locus. This latter piece of evidence is further supported by the fact that the temperature we derived from the 2MASS colors is the same as that derived by the ASPCAP pipeline (see Section 2). The ASPCAP pipeline fits model spectra to the continuumnormalized observed spectrum and is, importantly, independent

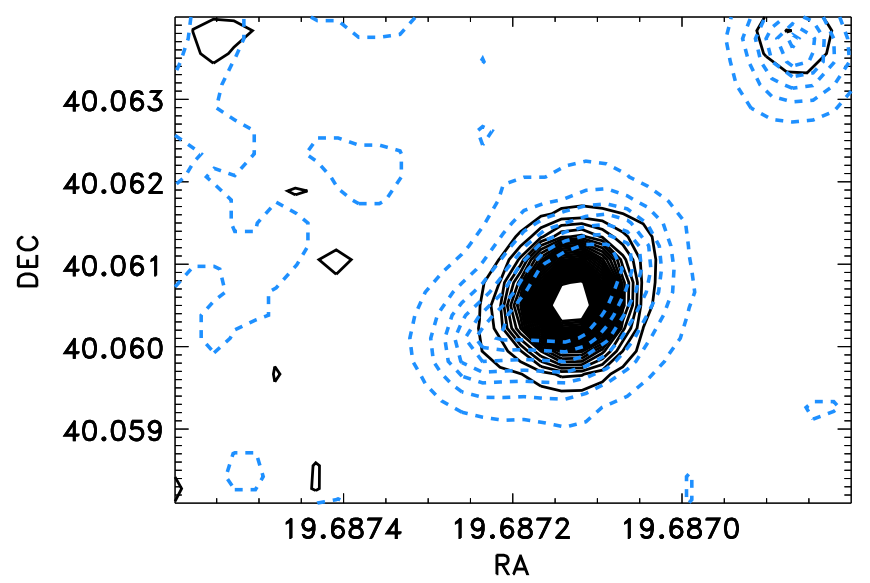

Figure 6. Image of the Li-rich star in 2MASS $H$-band image (solid lines) and the DSS optical blue image (dashed lines). The optical image of the Li-rich star is slightly elongated on one side compared to the infrared image.

of any assumption of reddening. Therefore, the ASPCAP temperature verifies that the reddening of the Li-rich star is the same as the rest of the cluster and thus shares the temperature and magnitude of the cluster's isochrone.

\subsubsection{A Deeper Look at Proper Motions}

One of the major pieces of evidence against membership for the Li-rich star is that Platais et al. (2013) find proper motions inconsistent with membership. However, the authors of that paper noted that finding low proper motion membership probabilities for stars with high RV membership probabilities is likely due to source confusion (see their Figure 6). In fact, an earlier proper motion study (Sanders 1972) gave the Li-rich star (star 90 in that paper) a $90 \%$ membership probability. An inspection of a Digitized Sky Surveys (DSS) optical blue image and the 2MASS $H$-band image in Figure 6 reveals an elongation in the optical DSS image that is not present in the $\mathrm{H}$ band image. This is suggestive of a faint, blue source. If this source is visible in the plates used by Platais et al. (2013), or worse is only visible in some of the plates, it could shift the apparent photocenter of the Li-rich star and skew the proper motion measurement. ${ }^{27}$ Star 90 on the Sanders (1972) plate is very round, suggesting that the nearby blue source is too faint to adversely affect the earlier proper motion measurement. Indeed, the $V$ limit of the Sanders (1972) plate is quoted as $\sim 14.5 \mathrm{mag}$, whereas the magnitude limits of the plates used in Platais et al. (2013) are $V=17-22$ mag. Because this visual companion is faint and blue, it will not contribute significantly to the $H$-band spectrum of the Li-rich star, consistent with our analyses in Section 6.

\subsubsection{Problems with Replenishment Mechanisms}

Cluster membership adds more problems to both the internal and external replenishment models. As a cluster member, the evolutionary stage of the Li-rich star is clearly below the luminosity bump, which led A13 to favor the recent D12 models. However, there is a discrepancy between the D12 models and the Li-rich star's CMD position. D12's model predicts an evolution that traces an extended luminosity bump-like zigzag on the CMD. Unlike the luminosity bump, the extended zigzag in the

\footnotetext{
${ }^{27}$ See Note added in proof.
} 
D12 models parallels the first ascent RGB at lower luminosity. For a given $T_{\text {eff }}$, the $\Delta \log L$ is $\approx-0.2$, corresponding to a magnitude difference of +0.5 . The Li-rich star does not sit 0.5 magnitudes below the RGB in either optical or infrared CMDs. For external replenishment models, the other cluster RGs provide a revised estimate for pre-engulfment $A(\mathrm{Li})$, which is lower than that estimated in Section 7.1.3. Additionally, with a well known evolutionary stage, we can more accurately pinpoint a MESA model to estimate the size of the convection zone. Using the $1.7 M_{\odot}$ MESA run, we inspected the internal profiles for four evolutionary stages on the first ascent RGB near $T=4700 \mathrm{~K}$. In all cases, there is $\sim 1.4 M_{\odot}$ in the convective envelope, requiring $0.15 M_{\odot}$ of accreted material to explain the observed $A(\mathrm{Li})$.

\subsubsection{An Unusually Low Mass for the Li-rich Star}

Another implication of the cluster membership is that it allows us to put stronger credence on the asteroseismology result, since our spectroscopic $\log g$ agrees with the astero seismic $\log g$. The scaling relations for $\nu_{\max }$ and $\Delta \nu$ can both be cast to depend only on $T_{\text {eff }}, M$, and either $R$ or $L$. Since $T_{\text {eff }}$ is accurately known and has the weakest influence on $\nu_{\max }$ and $\Delta \nu$, the remaining quantities can be derived without any other assumptions. These results are given in the bottom section of Table 1. There is good agreement between the luminosity derived through asteroseismology and that derived assuming the star is in the cluster. This analysis places the Li-rich star at roughly the distance of the cluster but with a mass of only $0.7 M_{\odot}$, which explains the "low" $\log g$. The main sequence lifetime of such a low mass star exceeds the age of the universe. If this mass is correct, the most plausible explanation is that the star lost a significant fraction of its mass, and we suggest that this mass loss is related to the source of Li. Furthermore, this low mass does not nullify the CMD position as evidence of cluster membership. The luminosity of stars with H-burning shells is dictated by the mass of the stellar core, leading to a core mass-luminosity relationship. Boothroyd \& Sackmann (1988) demonstrated that this relationship holds for intermediate mass stars and that the total mass has a negligible effect on this relationship. Thus, if the Li-rich star began its life with the same mass as the other RGs and only lost mass recently, it should have the same core mass as the other RGs.

\subsubsection{External Enrichment at Low Mass}

The case for enrichment by an external source of $A(\mathrm{Li})$ is much more feasible if the star is really $0.7 M_{\odot}$. We can again adopt $A(\mathrm{Li})<0.7$ dex for the pre-engulfment abundance and use the MESA models of the $1.7 M_{\odot}$ star. If an engulfed companion strips $1 M_{\odot}$ of material from the star before it disperses into the remaining stellar envelope, then the remaining envelope is only $0.4 M_{\odot}$. Since the star is not entirely stripped of the original convective envelope, the mass loss not does extend deep enough to expose material with low ${ }^{12} \mathrm{C} /{ }^{13} \mathrm{C}$. Only a $45 M_{\text {Jup }}$ object is required to enrich $A(\mathrm{Li})$ up to the observed value. This mass is safely in the substellar companion regime of objects, which retain their birth $A(\mathrm{Li})$.

\subsubsection{Updated Abundances at Low $\log g$}

If $2 \mathrm{M} 19411367+4003382$ 's $\log g$ is truly so low, then we must re-derive the abundances. Because using the abundance sensitivities in Table 3 requires extrapolating by $0.2 \mathrm{dex}$ beyond the $\log g$ range we tested, we instead calculate all of the

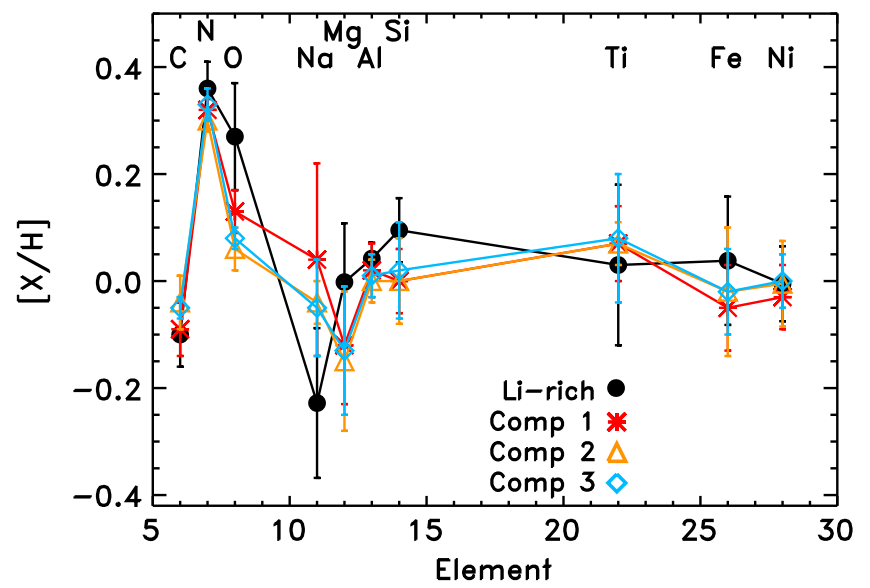

Figure 7. Elemental abundances normalized to solar (Asplund et al. 2009). The Li-rich star's abundances are computed for a $T_{\text {eff }}=4700 \mathrm{~K}$ and $\log g$ $=2.35 \mathrm{dex}$ atmosphere model. The abundances of the control stars are the same as in Figure 4.

new abundances with the lower $\log g$ atmosphere. We calculate the equivalent widths needed to create the abundances we measured for the Li-rich star with the $\log g=2.80$ dex model and then find how the abundances change for these same equivalent widths with a $\log g=2.35$ dex model. For the $\mathrm{C}, \mathrm{N}$, and $\mathrm{O}$ abundances from the molecular lines, we iterate the process until the output abundances match the input abundances. The lower $\log g$ has the following effects: Fe changes by $+0.09, \mathrm{C}$ by $-0.15, \mathrm{~N}$ by +0.08 , $\mathrm{O}$ by +0.02 , $\mathrm{Na}$ by +0.03 , $\mathrm{Mg}$ by +0.16 , $\mathrm{Al}$ by +0.17 , Si by +0.11 , Ti by +0.05 , and $\mathrm{Ni}$ by -0.005 dex. Figure 7 shows these new abundances compared to those of the comparison stars. The updated $A(\mathrm{Al})$ is now in excellent agreement with the other cluster stars. The rather large increases in the abundances of $\mathrm{Mg}, \mathrm{Si}$, and $\mathrm{Fe}$ are still consistent with the abundances of the other cluster stars within the uncertainties. Furthermore, the $\mathrm{C} / \mathrm{N}$ ratio is now 1.4 , which is also in much better agreement with other RGs in both NGC 6819 and in other clusters (Figure 3). However, oxygen is still unusually high, with $[\mathrm{O} / \mathrm{Fe}] \sim 0.23 \pm 0.15$ dex.

\subsubsection{Comparison to Other Known Li-rich Cluster Giants}

Finally, if $2 \mathrm{M} 19411367+4003382$ is truly a member of NGC 6819, then we can compare this star to the few other known Li-rich RGs in other open clusters. These include Trumpler 5 (Tr 5) \#3416 (Monaco et al. 2014) and Berkeley 21 (Be 21) T33 (Hill \& Pasquini 1999). Both of these open clusters have significantly sub-solar metallicities, near -0.5 dex. In both cases, the Li-rich stars are super Li-rich, $A(\mathrm{Li})>3.3$ dex. $\operatorname{Tr} 53416$ is also known to be a slow rotator and have ${ }^{12} \mathrm{C} /{ }^{13} \mathrm{C}=14$. The Li-rich star in $\operatorname{Tr} 5$ sits on the $\mathrm{RC}$, while the star in Be 21 sits a magnitude above the RC. Therefore, 2M19411367+4003382 does not share many common properties with these other known Li-rich stars in open clusters. It is of solar metallicity, fainter than its host cluster's RC, not super Li-rich, and has faster rotation and higher ${ }^{12} \mathrm{C} /{ }^{13} \mathrm{C}$ than the $\operatorname{Tr} 5$ Li-rich star. Additionally, 2M19411367+4003382's initial mass was presumably $1.7 M_{\odot}$, and Monaco et al. (2014) estimates that the masses of the $\operatorname{Tr} 5 \mathrm{RC}$ stars are 1.0-1.4 $M_{\odot}$, while Bragaglia $\&$ Tosi (2006) gives $1.4 M_{\odot}$ as the mass of the turn-off stars in $\mathrm{Be} 21$. 


\section{CONCLUSIONS}

We have performed a spectroscopic analysis of the Li-rich star 2M19411367+4003382, and we have provided crucial measurements of $\log g, \mathrm{C} / \mathrm{N}$, and ${ }^{12} \mathrm{C} /{ }^{13} \mathrm{C}$ that confirm that the star is an evolved $\mathrm{RG}$ with evidence of having completed FDU (in its $\mathrm{C} / \mathrm{N}$ and ${ }^{12} \mathrm{C} /{ }^{13} \mathrm{C}$ ) but showing no evidence of low ${ }^{12} \mathrm{C} /{ }^{13} \mathrm{C}$ that should accompany the previously favored model of explaining the star's enriched Li. We have also measured abundances of eight additional elements and found a chemical similarity between the Li-rich star and the other cluster members in elements that are not affected by nuclear processing, including both iron group $(\mathrm{Fe}, \mathrm{Ni})$ and $\alpha$ elements $(\mathrm{Mg}, \mathrm{Si}, \mathrm{Ti})$.

The membership of 2M19411367+4003382 has recently been questioned by both asteroseismic and recent proper motion measurements. We have argued that an older proper motion measurement may be more reliable, and this older measurement is compatible with membership. The asteroseismic non-membership evidence actually contains a surprising result. The asteroseismic $\nu_{\max }$ yields a $\log g$ lower than that expected for members, which we confirm spectroscopically. Recomputing the abundances at lower $\log g$ brings the abundances of the Li-rich star in better agreement with the other RGs in NGC 6819. Furthermore, $\nu_{\max }$ and $\Delta \nu$ also give the star's luminosity and mass and place the Li-rich star at roughly the distance to the cluster, but with a mass of only 0.7 $M_{\odot}$. Such a low mass star could not have evolved to the RG stage, implying that the Li-rich star has lost significant mass. The similar luminosity to other NGC 6819 RGs implies that the Li-rich star has a similar core mass to the other RGs, which is also consistent with the picture of extensive mass loss. For the case of external enrichment, this low stellar mass is the only case where a putative engulfed companion can have low enough mass to have retained its birth $\mathrm{Li}$ but still be massive enough to contribute enough $\mathrm{Li}$ to explain the observed stellar $A(\mathrm{Li})$. A more detailed analysis of the stellar oscillation spectrum may provide new insights on whether the scaling relationships do not apply to this star or whether the internal structure of the Li-rich star can shed light on its anomalous Li abundance and mass.

We thank the anonymous referee for providing valuable feedback on our manuscript. J.K.C. acknowledges partial support for this work by an appointment to the NASA Postdoctoral Program at the Goddard Space Flight Center, administered by Oak Ridge Associated Universities through a contract with NASA. V.S. acknowledges partial support for this research from the National Science Foundation (AST1109888). K.G.S acknowledges support from NSF PAARE grant AST-1358862. S.R.M. acknowledges support from NSF grants AST 1109718 and AST 1312863. Funding for SDSS-III has been provided by the Alfred P. Sloan Foundation, the Participating Institutions, the National Science Foundation, and the U.S. Department of Energy Office of Science. The SDSS-III web site is http://sdss3.org/. SDSS-III is managed by the Astrophysical Research Consortium for the Participating Institutions of the SDSS-III Collaboration including the University of Arizona, the Brazilian Participation Group, Brookhaven National Laboratory, Carnegie Mellon University, University of Florida, the French Participation Group, the German Participation Group, Harvard University, the Instituto de Astrofisica de Canarias, the Michigan State/Notre Dame/
JINA Participation Group, Johns Hopkins University, Lawrence Berkeley National Laboratory, Max Planck Institute for Astrophysics, Max Planck Institute for Extraterrestrial Physics, New Mexico State University, New York University, Ohio State University, Pennsylvania State University, University of Portsmouth, Princeton University, the Spanish Participation Group, University of Tokyo, University of Utah, Vanderbilt University, University of Virginia, University of Washington, and Yale University. This publication uses data products both from the Wide-field Infrared Survey Explorer, which is a joint project of the University of California, Los Angeles, and the Jet Propulsion Laboratory California Institute of Technology, funded by the National Aeronautics and Space Administration, and from the Two Micron All Sky Survey, which is a joint project of the University of Massachusetts and the Infrared Processing and Analysis Center/California Institute of Technology, funded by the National Aeronautics and Space Administration and the National Science Foundation. The Digitized Sky Surveys were produced at the Space Telescope Science Institute under U.S. Government grant NAG W-2166. The images of these surveys are based on photographic data obtained using the Oschin Schmidt Telescope on Palomar Mountain and the UK Schmidt Telescope. The plates were processed into the present compressed digital form with the permission of these institutions.

Note added in proof. I. Platais (2015, private communication) pointed out that the separation of the faint blue source (at $V=16.69$ and 3.3 arcsec separation, AT13) is sufficiently large that it is unlikely to be affecting the astrometric measurements of the Li-rich star. Instead, the large proper motion errors of the Li-rich star are likely due to its position near the edge of the highest $\mathrm{S} / \mathrm{N}$ plates, obtained with the 200inch Hale telescope, where coma effects from the telescope's optics are large.

\section{REFERENCES}

Ahn, C. P., Alexandroff, R., Allende Prieto, C., et al. 2014, ApJS, 211, 17 Allende Prieto, C., Majewski, S. R., Schiavon, R., et al. 2008, AN, 329, 1018 Anthony-Twarog, B. J., Deliyannis, C. P., Rich, E., \& Twarog, B. A. 2013, ApJL, 767, L19

Asplund, M., Grevesse, N., Sauval, A. J., \& Scott, P. 2009, ARA\&A, 47, 481

Basu, S., Grundahl, F., Stello, D., et al. 2011, ApJL, 729, L10

Bertelli, G., Girardi, L., Marigo, P., \& Nasi, E. 2008, A\&A, 484, 815

Bertelli, G., Nasi, E., Girardi, L., \& Marigo, P. 2009, A\&A, 508, 355

Bessell, M. S., Castelli, F., \& Plez, B. 1998, A\&A, 333, 231

Boothroyd, A. I., \& Sackmann, I.-J. 1988, ApJ, 328, 641

Bragaglia, A., Carretta, E., Gratton, R. G., et al. 2001, AJ, 121, 327

Bragaglia, A., \& Tosi, M. 2006, AJ, 131, 1544

Carlberg, J. K., Cunha, K., Smith, V. V., \& Majewski, S. R. 2012, ApJ, 757, 109

Carlberg, J. K. 2014, AJ, 147, 138

Carr, J. S., Sellgren, K., \& Balachandran, S. C. 2000, ApJ, 530, 307

Charbonnel, C., \& Balachandran, S. C. 2000, A\&A, 359, 563

Cunha, K., Smith, V. V., Johnson, J. A., et al. 2015, ApJL, 798, L41

Denissenkov, P. A. 2012, ApJL, 753, L3

Eggleton, P. P., Dearborn, D. S. P., \& Lattanzio, J. C. 2008, ApJ, 677, 581

Eisenstein, D. J., Weinberg, D. H., Agol, E., et al. 2011, AJ, 142, 72

Gilroy, K. K. 1989, ApJ, 347, 835

González Hernández, J. I., \& Bonifacio, P. 2009, A\&A, 497, 497

Gunn, J. E., Siegmund, W. A., Mannery, E. J., et al. 2006, AJ, 131, 2332

Hill, V., \& Pasquini, L. 1999, A\&A, 348, L21

Hole, K. T., Geller, A. M., Mathieu, R. D., Platais, I., Meibom, S., \& Latham, D. W. 2009, AJ, 138, 159

Kjeldsen, H., \& Bedding, T. R. 1995, A\&A, 293, 87

Kumar, Y. B., Reddy, B. E., \& Lambert, D. L. 2011, ApJL, 730, L12

Luck, R. E. 1994, ApJS, 91, 309

Majewski, S. R. 2012, American Astronomical Society Meeting Abstracts, \#219 
Marigo, P., Girardi, L., Bressan, A., Groenewegen, M. A. T., Silva, L., \& Granato, G. L. 2008, A\&A, 482, 883

Meszaros, Sz., Holtzman, J., García Pérez, A. E., et al. 2013, AJ, 146, 133

Mikolaitis, Š., Tautvaišienè, G., Gratton, R., Bragaglia, A., \& Carretta, E. 2010 MNRAS, 407, 1866

Mikolaitis, Š., Tautvaišienè, G., Gratton, R., Bragaglia, A., \& Carretta, E. 2011a, MNRAS, 413, 2199

Mikolaitis, Š., Tautvaišienė, G., Gratton, R., Bragaglia, A., \& Carretta, E. 2011b, MNRAS, 416, 1092

Mikolaitis, Š., Tautvaišienè, G., Gratton, R., Bragaglia, A., \& Carretta, E. 2012, A\&A, 541, 137

Monaco, L., Boffin, H. M. J., Bonifacio, P., et al. 2014, A\&A, 564, LL6

Nidever, D. L., Hutzman, J. A., Allende Prieto, C., et al. 2015, AJ, submitted (arXiv:1501.03742)

Paxton, B., Cantiello, M., Arras, P., et al. 2013, ApJS, 208, 4

Pinsonneault, M. H., Elsworth, Y., Epstein, C., et al. 2014, ApJS, 215, 19
Platais, I., Gosnell, N. M., Meibom, S., et al. 2013, AJ, 146, 43 Sanders, W. L. 1972, A\&A, 19, 155

Schlegel, D. J., Finkbeiner, D. P., \& Davis, M 1998, ApJ, 500, 525

Skrutskie, M. F., Cutri, R. M., Stiening, R., et al. 2006, AJ, 131, 1163

Smiljanic, R., Gauderon, R., North, P., et al. 2009, A\&A, 502, 267

Smith, V. V., Cunha, K., Shetrone, M. D., et al. 2013, ApJ, 765, 16

Sneden, C. 1973, ApJ, 184, 839

Stello, D., Meibom, S., Gilliland, R. L., et al. 2011, ApJ, 739, 13

Tautvaišiene, G., Edvardsson, B., Tuominen, I., \& Ilyin, I. 2000, A\&A, 360,499

Tautvaišienè, G., Edvardsson, B., Puzeras, E., \& Ilyin, I. 2005, A\&A, 431, 933

Wood, M. P., Lawler, J. E., \& Shetrone, M. D. 2014, ApJL, 787, L16

Wright, E. L., Eisenhardt, P. R. M., Mainzer, A. K., et al. 2010, AJ, 140,1868

Zasowski, G., Johnson, J. A., Frinchaboy, P. M., et al. 2013, AJ, 146, 81

Zucker, S., \& Mazeh, T. 1994, ApJ, 420, 806 